\title{
The Gamma-Ray Distribution From Oriented Cerium-141
}

\author{
J. F. Schooley, ${ }^{1}$ D. D. Hoppes, and A. T. Hirshfeld
}

(March 2, 1962)

\begin{abstract}
The distribution of gamma-radiation emitted in the decay of $\mathrm{Ce}^{141}$ nuclei alined in neodymium ethylsulfate single crystals has been remeasured for temperatures as low as $0.0162{ }^{\circ} \mathrm{K}$ and has been found to be considerably more anisotropic than previcusly thought. The discrepancy is explained on the basis of preferential scattering of the plane-polarized radiation in the Dewar system. Similar measurements with $\mathrm{Ce}^{141}$ nuclei oriented in a polarized cerium ethylsulfate crystal have been made. The hyperfine constant for $\mathrm{Ce}^{141}$ in the neodymium ethylsulfate lattice is deduced as $A=0.0305 \pm 0.003 \mathrm{~cm}^{-1}$, and the $E 2 / M 1$ ratio for the 145 -kev gamma ray in $\operatorname{Pr}^{141}$ is $+0.068 \pm 0.008$.
\end{abstract}

\section{Introduction}

In order to use the gamma-ray distribution $[1]^{2} W(\theta)=1+\Sigma A_{\nu} f_{\nu} P_{\nu}(\cos \theta)$ from alined or polarized $\mathrm{Ce}^{141}$ as a secondary thermometer in experiments involving beta-ray distributions [2] or heat conduction in rare earth ethylsulfate crystals, we have reexamined this distribution experimentally. We find a considerably greater anisotropy than that reported by previous investigators [3, 4], and we find that scattering may offer an explanation for the discrepancy. Moreover, we are able to make an experimental determination of the hyperfine constants for cerium isotopes in concentrated neodymium ethylsulfate. Experiments with $\mathrm{Ce}^{141}$ in concentrated cerium ethylsulfate show that this system can produce almost total alinement when a small magnetic field is applied.

\section{Experimental Procedure}

\subsection{General}

The $\mathrm{Ce}^{141}$ used in this investigation was fissionproduct material which contained $\mathrm{Ce}^{144}$ and $\operatorname{Pr}^{144}$ as impurities. Conversion electron spectra observed with a thick-lens beta-ray spectrometer were used, together with data on the conversion coefficients [5], to find the contribution of the $134-\mathrm{kev} \mathrm{Ce}^{144}$ gammaray to the experimental spectrum of the $145-\mathrm{kev}$ gamma-ray of $\mathrm{Ce}^{141}$.

Approximately 1 microcurie of the activity was grown into each of four single crystals, each weighing 2 to 3 g. Two crystals contained only neodymium ethylsulfate and the activity. In another, 1 percent of the neodymium was replaced by stable cerium. The fourth crystal was concentrated cerium ethylsulfate. An inactive layer was grown around each crystal.

Although the experimental procedure used with the cerium ethylsulfate crystal was somewhat different from that used with the other specimens, the

1 National Academy of Sciences-National Research Council Postdoctoral Resident Research Associate.

2 Figures in brackets indicate the literature references at the end of this paper. gamma-ray detectors and the counting procedures were the same in each case. Three gamma-ray detectors, usually utilizing 2 in. long by 2 in. diam NaI cylinders, were mounted in a horizontal array into which the Dewar assembly was swung after adiabatic demagnetization of the specimen. Each detector was monitored by a single-channel pulse height analyzer, whose output was switched at $1 \mathrm{~min}$ intervals from one to another of a pair of scalers. The output of one detector was also recorded by a 100-channel pulse height analyzer during the orientation period. After a counting time of from 3 to $10 \mathrm{~min}$, the sample was warmed to the helium bath temperature in order to take a similar sampling of the isotropic radiation for normalization. The ratio of the "cold" to "warm" counting rates was then corrected for background, attenuation due to the finite solid angle subtended by the detector, and the gamma ray impurity.

\subsection{Neodymium Ethylsulfate Host Crystals}

The cryogenic apparatus used for the three neodymium ethylsulfate crystals has been described in detail by de Klerk and Hudson [6]; a useful modification has been the use of $\mathrm{He}^{3}$ exchange gas, i.e. gas introduced to provide thermal contact between the sample and the pumped $\mathrm{He}^{4}$ bath during the magnetization portion of the cycle. Pumping off the $\mathrm{He}^{3}$ gas produced thermal isolation for demagnetization in approximately $3 \mathrm{~min}$, an order of magnitude improvement over the use of $\mathrm{He}^{4}$. Each crystal was mounted with the $c$-axis horizontal in order that the horizontal 22-kilogauss field could be applied for maximum cooling effect. The data of Meyer [7] were used to deduce the temperatures reached for the various values of magnetic field and bath temperature; in these runs, the final temperature varied between $0.1^{\circ} \mathrm{K}$ and $0.016{ }^{\circ} \mathrm{K}$. In general, two of the gammaray detectors were placed to receive radiation emitted parallel to the $c$-axis, and the third sampled radiation emitted perpendicular to this alinement axis. Warming of the specimen could be detected by change in the gamma-ray counting rate and by change in the paramagnetic susceptibility; there was, however, no systematic variation in the gamma-ray data during any counting period. Furthermore, the initial rate 
of warming from the lowest temperatures was difficult to assess by the mutual inductance bridge, since the perpendicular susceptibility appeared to change very little below $0.020^{\circ} \mathrm{K}$. For higher initial temperatures, the change in temperature during the "cold" counting period varied from about 5 percent at 0.026 ${ }^{\circ} \mathrm{K}$ to about 10 percent at $0.100{ }^{\circ} \mathrm{K}$.

\subsection{Cerium Ethylsulfate Host Crystals}

The experimental arrangement in this case was dictated by the observation of Johnson and Meyer [8], indicative of an antiferromagnetic transition, that the adiabatic demagnetization process results in a minimum temperature of a cerium ethylsulfate specimen when the high initial field is reduced to 500 to 600 gauss, rather than to zero field. For an initial field of 22 kilogauss applied along the crystalline $c$-axis and a parallel residual field of 550 gauss, the final specimen temperature is calculated to be within the range 0.003 to $0.010^{\circ} \mathrm{K}$. In order to achieve optimum gamma-ray counting conditions in the present experiment, this residual field was supplied by a superconducting solenoid immersed in the helium bath. This coil consisted of 1,850 turns of 0.003 in. diam formvar-coated niobium wire, wound in two layers on the glass wall of the inner space of a light-guide cryostat designed for beta-ray distribution studies [9]. Since this solenoid produced a vertical field of no more than 1,700 gauss, it was necessary to use the large horizontal-field magnet for the initial field. In order to apply each field, in turn, along the crystalline $c$-axis, advantage was taken of the highly anisotropic $g$-values of cerium ethylsulfate [10]. The sample was suspended by a nylon thread passing horizontally through the crystal slightly above its center of gravity so that with zero applied field the $c$-axis was maintained vertical. Application of the 22 -kilogauss field rotated the sample until the $c$-axis was horizontal. Upon demagnetization the crystal returned to the zero-field position, and it remained there when the solenoid was energized. The assembly was X-rayed under each of the above conditions to verify that the correct position was achieved.

As before, the gamma-ray counters were placed in a horizontal plane, which in this case corresponded to $\theta=90^{\circ}$ for each. Contrary to the observations with the neodymium ethylsulfate samples, the gamma-ray counting rate changed noticeably during succeeding 1-min periods, necessitating an extrapolation of the "cold" counting rate to the time of demagnetization, approximately $1 \mathrm{~min}$ before the start of counting.

\section{Results}

The angular dependence of the gamma-ray intensity from cerium-141 alined in neodymium ethylsulfate was measured at approximately $0.0162{ }^{\circ} \mathrm{K}$ and found to be satisfactorily represented by the function

$$
W(\theta)=1+(0.205 \pm 0.010) P_{2}(\cos \theta)
$$

$$
+(0.006 \pm 0.018) P_{4}(\cos \theta)
$$

where $\theta$ denotes the angle between the axis of alinement (in this case the crystalline $c$-axis) and the direction of gamma-ray emission. The coefficient of $P_{4}(\cos \theta)$ relative to that of $P_{2}(\cos \theta)$ can be calculated approximately, given an estimate of $f_{2}$; this ratio, $A_{4} f_{4} / A_{2} f_{2}$, is calculated to be 0.024 .

The temperature dependence of $\mathrm{W}\left(0^{\circ}\right)$ for the neodymium ethylsulfate crystals is shown in figure 1 . The data taken with the 1 percent cerium crystal could not be distinguished from that taken with the two essentially pure neodymium ethylsulfate crystals, and therefore it is also included in figure 1 . The error flags denote the standard deviation of the mean of a number of 5 to $10 \mathrm{~min}$ counts at a given temperature. Overall, some one hundred such counts were made on the three samples. Corrections for detector solid angle, background, and cerium-144 impurity amounted to an average of 10 percent of $A_{2} f_{2}$. The temperature scale was derived from the data of H. Meyer [7] for a magnetic field applied parallel to the crystalline $c$-axis.

The data of Cacho et al., [3] were taken with low specific activity cerium-141, so that the sample corresponded almost exactly with the present 1 percent cerium crystal. Since $A_{2} f_{2}$ was plotted by them as a function of $1 / T^{*}$, approximate corrections for the nonspherical shape of the specimen and for Meyer's $T-T^{*}$ (sphere) relation have been made. The adjusted data, shown in figure 1 , are in agreement with similar corrections made by Scurlock [11]. The triangular point in figure 1 represents the value of $A_{2}$ calculated from the experiments of Ambler, Hudson, and Temmer [4], using planar alinement of cerium-141 in cerium magnesium nitrate, multiplied by the $f_{2}$ deduced from the present experiments for the temperature $0.0166^{\circ} \mathrm{K}$.

An interesting feature of the gamma-ray distribution was the change in $W(\theta)$ with decreasing energy of the detected photon. The spectrum measured at $0.016{ }^{\circ} \mathrm{K}$ and an isotropic comparison spectrum taken $1{ }^{\circ} \mathrm{K}$ are shown in figure $2 . \quad W\left(0^{\circ}\right)$ was $1.205 \pm 0.010$ for photon energies between approximately 140 and $170 \mathrm{kev}$; between 140 and $125 \mathrm{kev}$, however, the intensity decreased uniformly until at $115 \mathrm{kev}$ it reached a minimum value of $0.80 \pm 0.04$. Correspondingly, $W\left(90^{\circ}\right)$ was $0.90 \pm 0.01$ at the photopeak energy, rising to a maximum of $1.10 \pm 0.05$ at approximately $115 \mathrm{kev}$. This reversal in the anisotropy at lower energies was demonstrated to be a scattering phenomenon by the use of a collimating system consisting of a $1 \mathrm{~cm}$ hole in a gold foil surrounding the vacuum jacket and tin-lined lead cones which were placed in front of the detector crystals. Multichannel analysis of the photon spectrum under these conditions showed no reversal of the anisotropy around $115 \mathrm{kev}$. It should be pointed out that most of the data shown in figure 1 were taken with single-channel analyzers and that, to avoid the attenuation due to this scattering effect, the discriminator thresholds were set at about $135 \mathrm{kev}$. This selection made the counting rate sensitive to small shifts in the pulse height, as is evidenced by the relatively large standard deviations shown on the data of figure 1 . 


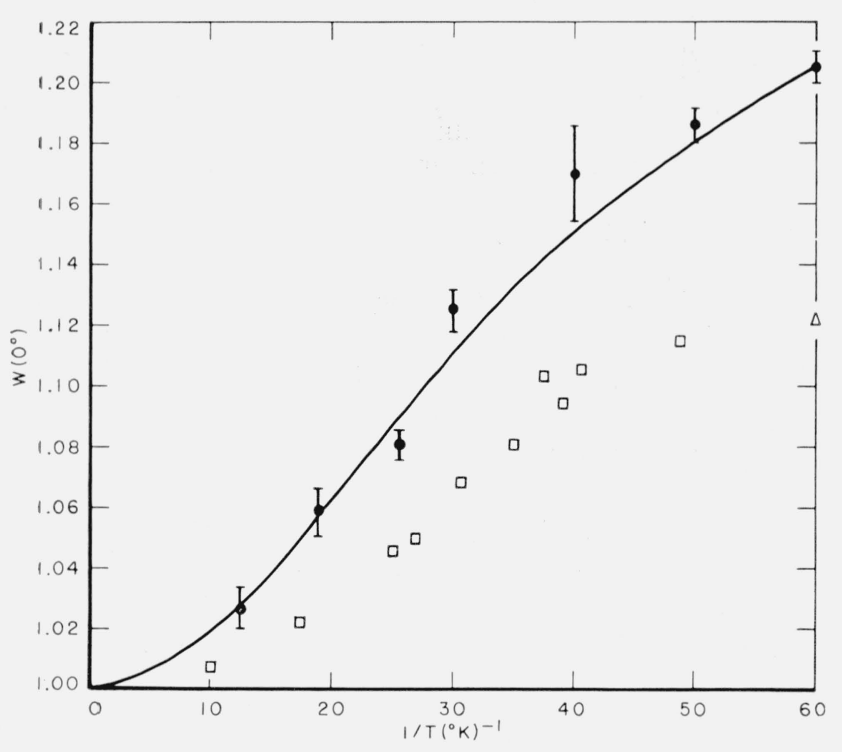

Figure 1. The temperature dependence of $\mathrm{W}\left(\mathrm{O}^{\circ}\right)$ (which equals the quantity $\left(1+\mathrm{A}_{2} \mathrm{f}_{2}\right)$ in this case) for $\mathrm{Ce}^{141}$ in neodymium ethylsulfate.

- present data;

Cacho et al. [3]

$\triangle$, calculated from data of Ambler, Hudson, and Temmer [4];

- theoretical curve (see discussion sec, 4).

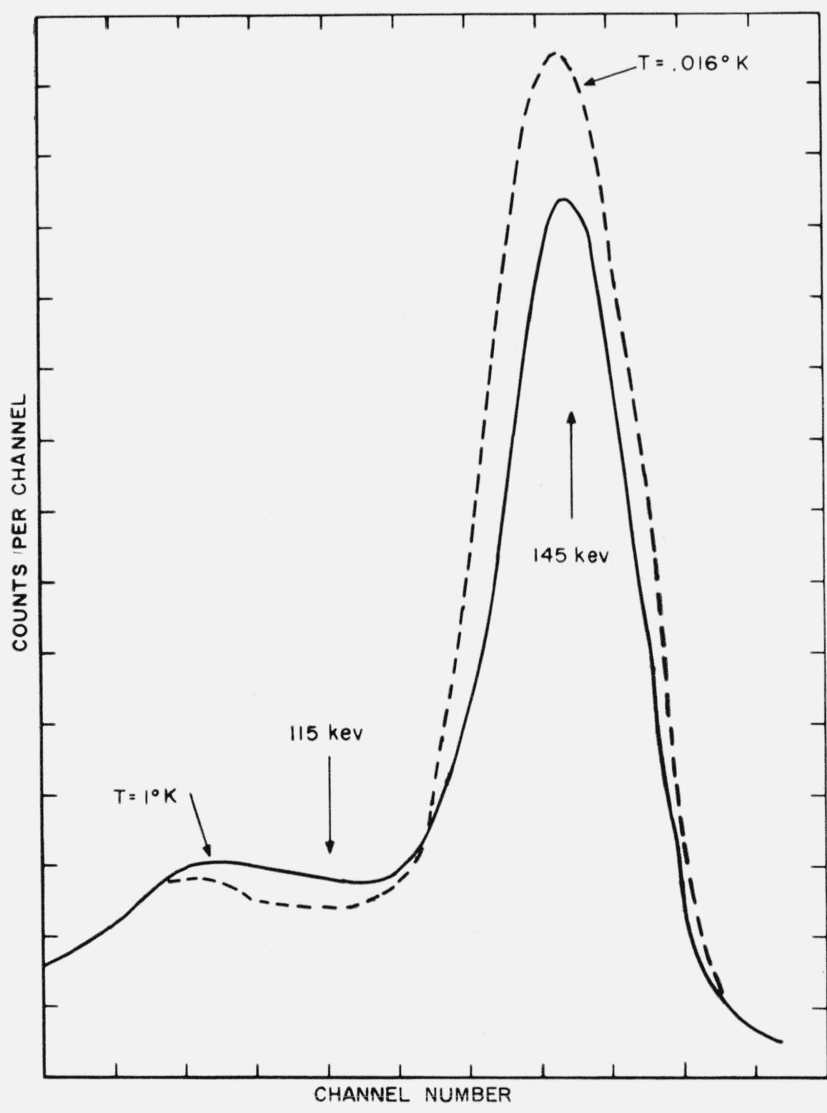

FIGURE 2. The energy spectrum of gamma-radiation from $\mathrm{Ce}^{141}$ in neodymium ethylsulfate observed at $\theta=0^{\circ}$ without collimation.

-, isotropic spectrum taken at $T=1^{\circ} \mathrm{K}$;

-., spectrum taken at $T=0.016^{\circ} \mathrm{K}$

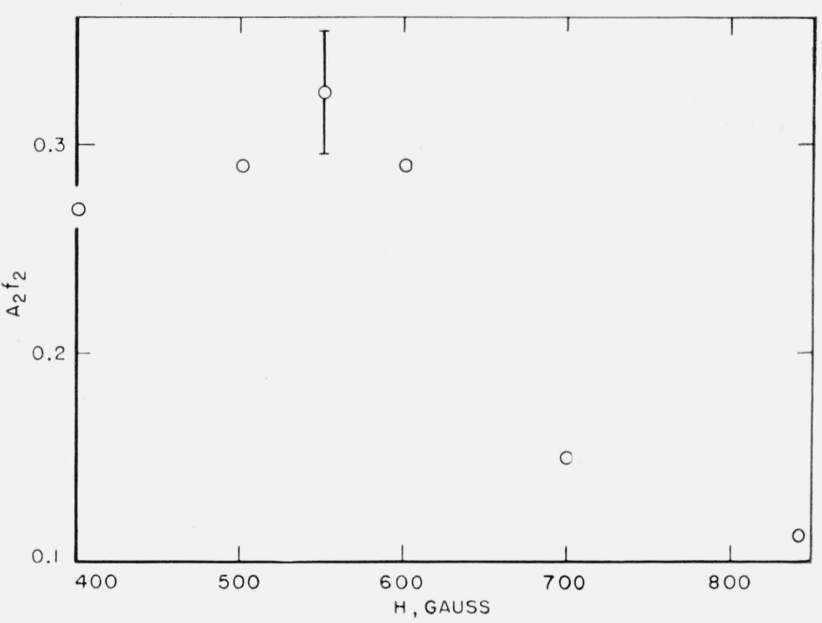

Figure 3. The coefficient of $\mathrm{P}_{2}(\cos \theta)$ for gamma-radiation from $\mathrm{Ce}^{141}$ oriented in cerium ethylsulfate, as a function of magnetic field applied parallel to the crystalline c-axis.

The results of the cerium ethylsulfate experiments are illustrated in figure 3 , in which the experimental $A_{2} f_{2}$ is plotted against applied field. The maximum value of $A_{2} f_{2}$ is seen to be about $0.325 \pm$ 0.03. The corresponding magnitude of $f_{2}$ can be estimated, assuming the hyperfine interaction to be similar to that deduced for neodymium ethylsulfate as the host lattice, to be nearly 0.571 , the value characteristic of complete alinement. The fact that $f_{2} \leqq 0.571$ allows a lower limit of 0.508 to be placed on $A_{2}$. This limit $A_{2}$ was used in the analysis of the neodymium ethylsulfate data that follows.

\section{Discussion}

The change in sign of the experimental anisotropy at energies below about $130 \mathrm{kev}$ can be accounted for by noting that the gamma-rays emitted from the alined cerium-141 are plane-polarized, and considering the effect of this polarization on subsequent scattering of the photons from the vacuum jacket and the Dewar walls (see fig. 4). With a typical (uncollimated) counting geometry the radiation de-

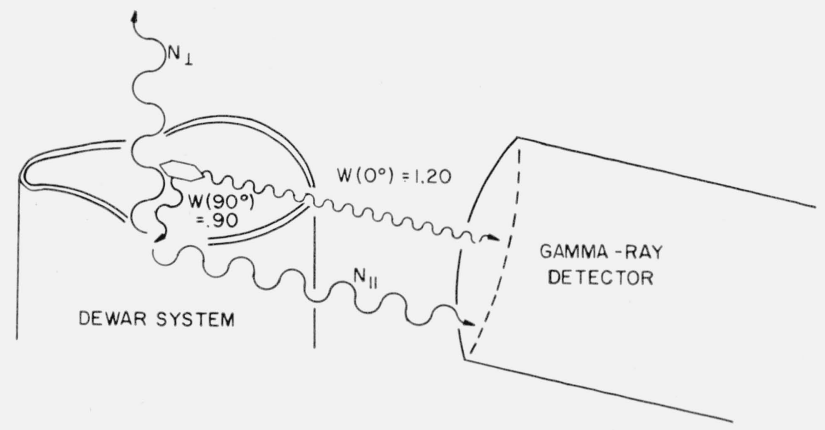

FIguRE 4. Schematic diagram of source, Dewar system, and detector placed at $\theta=0^{\circ}$.

[Radiation emitted in the $0=90^{\circ}$ direction and scattered by the Dewar system through an angle of $90^{\circ}$ can impinge on the uncollimated detector. Indicated values of
beams.] 
fined as $N_{\|}$[12], taking the scattering medium as the Dewar system, could impinge directly upon the detector placed along the axis of alinement. A photon beam of $145 \mathrm{kev}$ must be scattered through $90^{\circ}$ in order to be degraded to an average energy of $115 \mathrm{kev}$ according to the simple Klein-Nishina relation [13]; thus, without considering polarization of the gammarays, the ratio of "cold" to "warm" counting rates measured at $115 \mathrm{kev}$ energy is seen to be less than unity. The (cold/warm) counting rate for the scattered beam identified as $N_{\|}$in figure 4 would in fact be 0.90 , but taking account of the detector solid angle and energy resolution raises this ratio to about 0.94 . One can include the effect of preferential scattering of the radiation emitted at $\theta=90^{\circ}$ due to it plane-polarized nature by use of the measurement of $N_{\perp} / N_{\|}=1.58$ at $T=0.025^{\circ} \mathrm{K}$ [3]. In this case the value of $N_{\|}$, normalized to unity for isotropic, unpolarized radiation, is 0.78 [12]. This is in agreement with the experimental measurement at that energy. It is seen that for photons losing more or less energy than those included in $N_{\|}$, the normalized intensity would be nearer unity, again in accord with experiment. A similar argument holds for measurements made with the gamma-ray detector placed to receive radiation emitted perpendicular to the $c$-axis.

The scattering phenomenon takes on particular significance in the present experiment because the small energy loss in $90^{\circ}$ scattering places the "reverse anisotropy" region adjacent to the energy region of interest. Inclusion of some or all of this scattered radiation can be shown by multichannel analysis to reduce the measured value of $A_{2} f_{2}$ by a factor as high as 0.58. The discrepancy between the present data and that of the previous investigators [3] is of just this magnitude, and may in fact lie in the various gamma-ray pulse height analyzer settings. It might be pointed out that no attenuation factor for imperfections in the crystal lattice bas been taken account of in the analysis of the present data. As was observed before, the presence of 1 percent cerium in the neodymium ethylsulfate crystal was found to have no measurable effect on the alinement results.

From the data taken with the cerium ethylsulfate crystal, $\left(A_{2} f_{2}\right)_{\max }=0.32 \pm 0.03$. Since $f_{2}=0.571$ for complete alinement, $A_{2} \geq 0.30 / 0.57=0.52$. Assuming the zero-field Hamiltonian in neodymium ethylsulfate $\mathscr{H}=A S_{z} I_{z}+B\left(S_{x} I_{x}+S_{y} I_{y}\right)+C S_{z} T_{z}$, where $T_{z}$ is the total spin component of the two nearest neighbor cerium ions [14] and where $B \sim 0.05 A$, the energy levels and consequently the parameter $f_{2}(T)$ of the system can be calculated. From a comparison of the calculated temperature dependence of $f_{2}$ for for various values of the hyperfine parameter $A$ with the experimental $A_{2} f_{2}$ curve, taken with the limit on $A_{2}$ deduced from the cerium ethylsulfate data, the hyperfine constant $A=0.0305 \pm 0.003 \mathrm{~cm}^{-1}$. If one assumes that $A=$ (constant) $\mu_{N} / I$, then using the value of the nuclear magnetic moment of $\mathrm{Ce}^{141}$ determined by Kedzie et al., [15] corrected by use of the more accurate value of $\left\langle r^{-3}\right\rangle$ due to Judd and Lindgren [16], one obtains the value $0.091 \pm 0.01$ for the constant. The constant thus calculated is somewhat larger than the value calculated by Haag et al., [17] on the basis of the theory of Elliott and Stevens, [18] for cerium ethylsulfate.

The value of $f_{2}$ calculated at $T \sim 0.0162{ }^{\circ} \mathrm{K}$ is $0.414 \pm 0.025$; this number, taken with the experimental $A_{2} f_{2}$ at the same temperature yields for $A_{2}$ the value $0.495 \pm 0.03$. The calculated temperature dependence of $A_{2} f_{2}$ for $A_{2}=0.495$ and $A=0.0305 \mathrm{~cm}^{-1}$ is shown in figure 1. Taking the attenuating effect due to the unobserved beta decay to be represented by the factor 0.97 [19], we find for the $145 \mathrm{kev}$ gamma-ray of $\operatorname{Pr}^{141}$ an $E 2$ to $M 1$ mixing ratio [12] $\delta=+0.068 \pm 0.008$.

The authors wish to acknowledge the helpful suggestions of E. Ambler of the Cryogenic Physics Section.

Note: Since this paper was prepared, data on the gamma-ray distribution from $\mathrm{Ce}^{141}$ oriented in neodymium ethylsulfate has been noted in the thesis of J. N. Haag (UCRL 9872, September 29, 1961 (unpublished)). These data are in close agreement with the corresponding data of the present authors.

\section{References}

[1] See e.g., E. Ambler, Methods of nuclear orientation. Progress in Cryogenics, 1960 pp. 249, 265, and 268, $A_{\nu}=\frac{B_{\nu}}{f_{\nu}} \times$ weighted average of $U_{\nu}\left(L_{\beta}\right) \times F_{\nu}^{\prime}\left(L I_{f} I_{i}\right)$, in the notation of this author. The $U_{\nu}$ give the attenuation due to a beta transition of multipolarity $L_{\beta}$, while the $F_{\nu}^{\prime}$ involve the nuclear spins, vector coupling coefficients, and the gamma multipole mixing ratio, $\delta$

[2] D. D. Hoppes, Technical Note \#93, NBS Aug. 1961.

[3] C. F. M. Cacho, M. A. Grace, C. E. Johnson, A. C. Knipper, R. G. Scurlock, and R. T. Taylor, Phil. Mag. 46, 1287 (1955).

[4] E. Ambler, R. P. Hudson, and G. Temmer, Phys. Rev. 97, 1212 (1955).

[5] Nuclear Data Sheets, National Research Council, NRC 58-10-81 (National Academy of Sciences, Washington, D.C.)

[6] D. de Klerk and R. P. Hudson, J. Res. NBS 53, 173 (1954).

[7] H. Meyer, Phil. Mag. 2, 521 (1957).

[8] C. E. Johnson and H. Meyer, Proc. Roy. Soc. A253, 199 (1959).

[9] C. S. Wu, E. Ambler, R. W. Hayward, D. D. Hoppes, and R. P. Hudson, Phys. Rev. 105, 1414 (1957); R. W. Hayward and D. D. Hoppes, IRE Trans. on Nuclear Science (IRE, New York, 1958) NS5 No. 3.

[10] A. Bogle, A. H. Cooke, and S. Whitley, Proc. Phys. Soc. A 64, 931 (1951).

[11] Private communication from C. E. Johnson to E. Ambler (1960)

[12] See, for example, E. Ambler Methods of nuclear orientation, Progress in Cryogenics, 1960, p. 272.

[13] A. T. Nelms, NBS Circular 542, Aug. 28, 1953, p. 19.

[14] C. E. Johnson, J. F. Schooley, and D. A. Shirley, Phys. Rev. 120, 2108, (1960).

[15] R. W. Kedzie, M. Abraham, and C. D. Jeffries, Phys. Rev. 108, 54 (1957).

[16] B. R. Judd and I. Lindgren, Phys. Rev. 122, 1802 (1961).

[17] J. N. Haag, C. E. Johnson, D. A. Shirley, and D. H. Templeton, Phys. Rev. 121, 591 (1961).

[18] R. J. Elliot and K. W. H. Stevens, Proc. Rev. Soc. (London) A215, 437 (1952).

[19] R. W. Hayward, A. T. Hirshfeld, D. D. Hoppes, and R. S. Kaeser (to be published).

(Paper 66A4-165) 